\title{
The Origin and Limit of Asymmetric Transmission in Chiral Resonators
}

\author{
Nikhil Parappurath, ${ }^{\dagger}$ Filippo Alpeggiani, ${ }^{\dagger,+}$ L. Kuipers, ${ }^{\dagger, \dagger}$ and Ewold Verhagen ${ }^{*}{ }^{\dagger}$ \\ $\dagger$ Center for Nanophotonics, AMOLF, Science Park 104, 1098 XG Amsterdam, The Netherlands \\ ${ }^{\ddagger}$ Kavli Institute of Nanoscience, Department of Quantum Nanoscience, Delft University of Technology, P.O. Box 5046, 2600 GA \\ Delft, The Netherlands
}

\section{Supporting Information}

ABSTRACT: We observe that the asymmetric transmission (AT) through photonic systems with a resonant chiral response is strongly related to the far-field properties of eigenmodes of the system. This understanding can be used to predict the AT for any resonant system from its complex eigenmodes. We find that the resonant chiral phenomenon of AT is related to, and is bounded by, the nonresonant scattering properties of the system. Using the principle of reciprocity, we determine a fundamental limit to the maximum AT possible for a single mode in any chiral resonator. We propose and follow a design route for a highly chiral dielectric photonic crystal structure that reaches this fundamental limit for AT.

KEYWORDS: optical chirality, asymmetric transmission, nanophotonics, photonic crystals, coupled-mode theory

A strong chiral response is essential for realizing devices that can manipulate the polarization of light. Natural chiral materials rely on bulk properties including birefringence and result in thick and bulky devices for polarization control. Much stronger chirality can be realized by exploiting the interaction of light with artificial nanostructures. ${ }^{1-3}$ Such interactions are observed to be enhanced through local resonances such as those supported by plasmonic antennas, ${ }^{4,5}$ periodically structured dielectric waveguides, ${ }^{6}$ etc. Arrangements of subwavelength-sized optical scatterers, called metasurfaces, are known for their exotic light-steering properties and polarization-dependent response. ${ }^{7,8}$ A better understanding of lightmatter interaction at the nanoscale will help us to realize optical metasurfaces with designable vectorial near and far electromagnetic fields. Polarization-manipulating nanostructures are also important for realizing compact and/or on-chip polarization rotators, wave plates, and polarizing beam splitters. ${ }^{9-13}$

An extreme possible consequence of the chirality of a system is asymmetric transmission (AT), the difference in total transmittance when light with a certain polarization impinges from opposite sides of the system. ${ }^{14}$ While it is possible to realize systems that radiate asymmetrically in opposite directions by breaking mirror symmetry in the propagation direction, ${ }^{15}$ AT however requires a strongly chiral response.

Notably, when an emitter is placed in asymmetrically transmitting systems, this strong chirality also implies a significant difference between the polarizations of the emitted light in opposite sides of the system. Realization of AT in nanostructures thus relates directly to potential functionalities such as polarization control of spontaneous emission, ${ }^{16}$ spindependent light emission, ${ }^{17,18}$ and enantioselective sensing. ${ }^{19}$
There has been a considerable number of experimental attempts at realizing strong chirality in nanostructures. Several of these have been shown to offer AT for circularly polarized light using both metallic ${ }^{20-23}$ and dielectric ${ }^{24,25}$ structures. However, to realize AT for linearly polarized light is significantly more challenging, as it strictly requires broken mirror symmetry in the propagation direction. ${ }^{26}$ So far, attempts at realizing AT for linearly polarized light have been restricted mainly to metallic structures. ${ }^{27-31}$ Important open questions remain, such as how to introduce an efficient symmetry breaking, what is the maximum AT that can be achieved, and how to design structures that can offer this maximum AT.

In this work, we investigate in detail the AT for linearly polarized light in dielectric chiral resonators. We show that the quasinormal modes of the system can be used to predict the AT for any system. We develop a theoretical formalism to find structures that can offer very high AT. Comparing full-field simulations to original theory, the origin and limits of AT are explored in terms of the properties of the quasinormal modes of the system. We uncover an important relation between the AT, which is a resonant phenomenon, and the direct reflectivity, which is a nonresonant property of the system. This relation, derived from the principle of reciprocity, creates a fundamental limit for AT in any chiral resonator. Following an optimization strategy to conform to this result, an example design for a photonic crystal structure with subwavelength thickness that can offer AT as high as $84 \%$ is proposed.

Received: November 28, 2016

Published: March 29, 2017 


\section{ON THE ORIGIN OF AT IN RESONANT SYSTEMS}

Figure 1 illustrates the concept of AT in chiral systems. Linearly polarized light is normally incident on the system in the forward direction (top-to-bottom direction in the figure). In the figure, the polarization of the incident beam is $\hat{\mathbf{s}}_{\mathbf{1}}$. Let the orthogonal polarization component be called $\hat{\mathbf{s}}_{\mathbf{2}}$. The transmitted electric field of light can, in principle, contain both components. For $\hat{\mathbf{s}}_{1}$-polarized incident light with electric field $\mathbf{E}_{1}^{\text {inc }}$, the complex forward transmission coefficients corresponding to the two electric field components along $\hat{\mathbf{s}}_{1}$ and $\hat{\mathbf{s}}_{2}$ in the transmitted light can be defined as $t_{11}^{\mathrm{f}}=\left(\hat{\mathbf{s}}_{\mathbf{1}} \cdot \mathbf{E}^{\mathrm{tran}}\right) / \mathbf{E}_{1}^{\text {inc }}$ and $t_{21}^{\mathrm{f}}=$ $\left(\hat{\mathbf{s}}_{2} \cdot \mathbf{E}^{\text {tran }}\right) / \mathbf{E}_{1}^{\text {inc }}$, where $\mathbf{E}^{\text {tran }}$ is the total transmitted electric field. The respective transmittances, ratios of the transmitted intensities and the incident intensity, are then $T_{11}^{f}=\left|t_{11}^{f}\right|^{2}$ and $T_{21}^{f}=\left|t_{21}^{f}\right|^{2}$. The total transmittance in the forward direction for $\hat{\mathbf{s}}_{1}$-polarized incidence can then be expressed as $T_{1}^{f}=T_{11}^{f}+T_{21}^{f}$. Similarly, when $\hat{\mathbf{s}}_{1}$-polarized light is incident from the opposite side of the structure, the total transmittance in the backward direction can be written as $T_{1}^{\mathrm{b}}=T_{11}^{\mathrm{b}}+T_{21}^{\mathrm{b}}$. AT is the difference in the total transmittances in the two directions for polarized light and can be represented as AT $=\left|T_{1}^{f}-T_{1}^{\mathrm{b}}\right|$. It has been shown $^{26,31}$ that for reciprocal materials $\mathrm{AT}$ is equivalent to the difference between cross-polarization conversion between two orthogonally polarized incidences. Thus, we can also write AT as the difference in the orthogonal transmittances for two mutually perpendicular polarized incidences in a given direction. i.e.,

$$
\mathrm{AT}=\left|T_{12}^{\mathrm{f}}-T_{21}^{\mathrm{f}}\right|
$$

where $T_{i j}^{\mathrm{f}}=\left|\left(\hat{\mathbf{s}}_{i} \cdot \mathbf{E}^{\mathrm{tran}}\right) / \mathbf{E}_{j}^{\mathrm{inc}}\right|^{2}$.

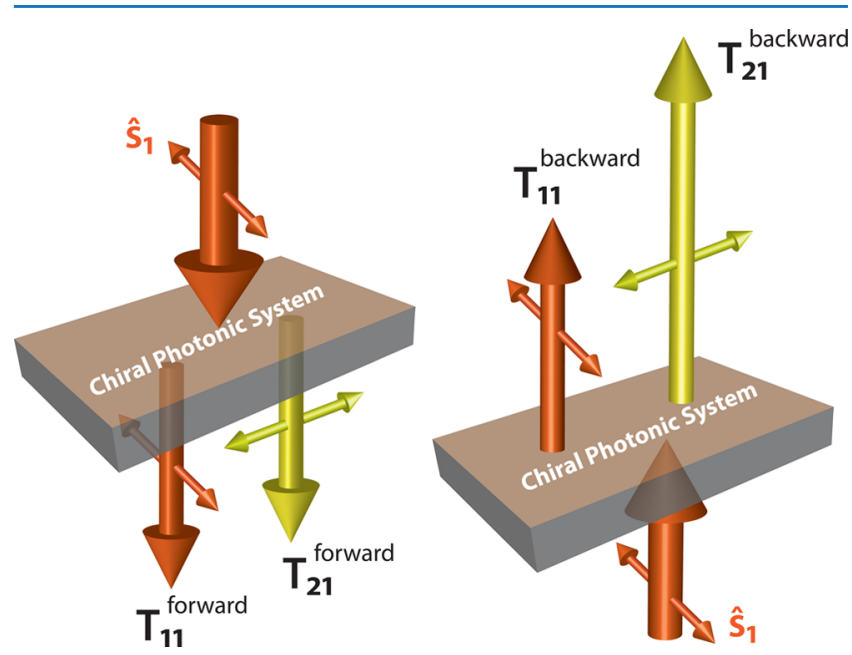

Figure 1. Concept of AT: Linearly polarized ( $\hat{\mathbf{s}}_{1}$-polarized) light is incident from the opposite sides of a chiral photonic system. $T_{11}$ and $T_{21}$ are the transmittances of the electric field component parallel and orthogonal to $\hat{s}_{1}$, respectively. AT is the difference between the total transmittances in the two directions.

In the following, we probe the origin of AT in chiral systems. Even though our conclusions and methodology apply to any general photonic systems, we will now illustrate them with a specific example, a dielectric bilayer photonic crystal slab. We consider light that propagates perpendicular to the plane in which the structure is periodic. A unit cell of the 2-D periodic structure is shown in the inset of Figure $2 \mathrm{~b}$. It is composed of two rectangular air holes stacked in mutually perpendicular
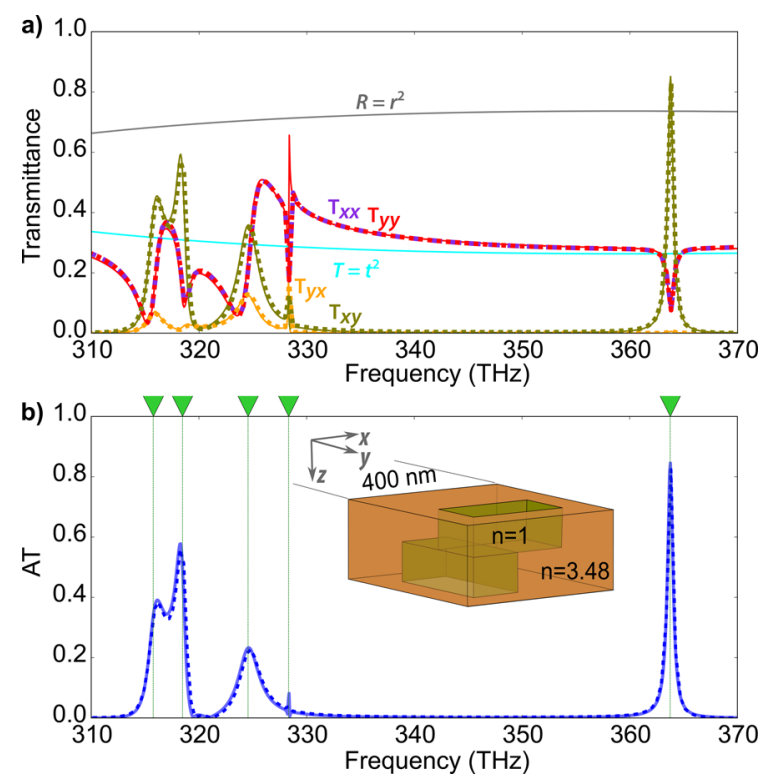

Figure 2. Transmission spectra: Various transmittances (a) and AT (b) as a function of frequency of the linearly polarized ( $x$ - or $y$ polarized) incident light in the positive $z$ direction. $T_{x x}, T_{y x}, T_{x y}, T_{y y}$, and AT are shown as violet, orange, olive, red, and dark blue, respectively. Thick dashed lines correspond to the FEM simulation data, and solid lines except gray and cyan feature transmittances predicted by our theoretical formalism. Gray and cyan lines represent the calculated background reflectance and transmittance, respectively, for an effective uniform slab (see the text in section 3). Green triangles represent the calculated real parts of the eigenfrequencies of the structure for zero parallel wave vector. The inset in panel b shows a unit cell of the proposed structure.

fashion in a high-index $(n=3.48)$ material. The orthogonal arrangement of holes breaks mirror symmetry in the propagation direction ( $z$-axis in the inset). Here, without loss of any generality, we choose $\hat{\mathbf{s}}_{\mathbf{1}}$ and $\hat{\mathbf{s}}_{\mathbf{2}}$ along the geometrical $x$ and $y$-axes, respectively. Finite element method (FEM) simulations ${ }^{32}$ are used to calculate various transmittances $T_{x x}$, $T_{y x}, T_{x y}$, and $T_{y y}$ of the structure in a given frequency range. In Figure $2 \mathrm{a}, T_{x x}$ and $T_{y x}$ (violet and orange dashed lines, respectively) are the simulated parallel and orthogonal transmittances for $x$-polarized incident field. $T_{x y}$ and $T_{y y}$ (red and olive dashed lines) denote the simulated orthogonal and parallel transmittances, respectively, for $y$-polarized incident field. The parallel transmittance for both incident polarizations, $T_{x x}$ and $T_{y y}$, coincides over the entire frequency range. However, there is a significant difference between the spectra of orthogonal transmittances $T_{y x}$ and $T_{x y}$ at certain frequencies. The AT spectrum for $x$ (or $y$ ) polarized incident light is calculated from the simulation results using eq 1 and shown as a dashed blue line in Figure $2 b$.

As evident from the figure, the AT has pronounced features around certain frequencies. The resonant nature of this chiral response hints at a connection between the response and resonant modes of the structure. The parallel component of the momentum is zero for light at normal incidence. Due to parallel momentum conservation, a normally incident wave would only excite modes with a wave vector having zero parallel component (i.e., $k_{\|}=0$, which corresponds to the $\Gamma$ point in momentum space). Using a commercial FEM mode solver, ${ }^{32}$ we calculate the eigenfrequencies (green triangles in Figure 2b) of the structure for a parallel wavevector of zero. These 
simulations reveal that the resonances in the AT spectrum indeed coincide with the real parts of the eigenfrequencies of the structure, indicating a strong relation between the AT and the complex-frequency modes of the structure. With this understanding of the origin of AT, we now develop a theoretical formalism to predict AT from the properties of these eigenmodes.

\section{PREDICTING AT FROM THE QUASINORMAL MODES OF A SYSTEM}

To qualitatively predict AT, we follow a general approach and develop a theoretical formalism based on coupled-mode theory. The theory explores the relation among incoming waves, outgoing waves, and guided modes in any chiral resonator.

Consider an open optical system having a set of quasinormal modes as shown in Figure 3. The modes interact with incoming

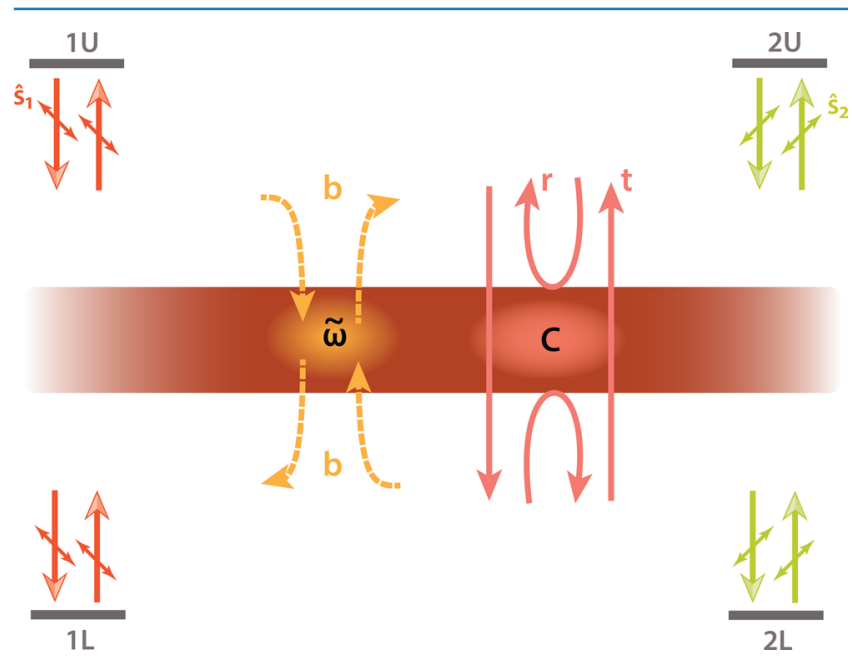

Figure 3. Coupled-mode-theory-based concept of eigenmodes' interaction with incoming/outgoing waves. Four ports, $1 \mathrm{U}, 2 \mathrm{U}, 1 \mathrm{~L}$, and $2 \mathrm{~L}$, represent incoming and outgoing electromagnetic plane waves of the two polarizations $\hat{\mathbf{s}}_{1}$ and $\hat{\mathbf{s}}_{2}$. Scattering eigenvector $\mathbf{b}$ mediates the interaction of resonances $(\tilde{\omega})$ with these ports. Waves can also couple directly $(C)$ through reflection and transmission coefficients $r$ and $t$. See the text for a detailed description.

and outgoing plane waves above and below the system. The two orthogonal polarizations $\hat{\mathbf{s}}_{1}$ and $\hat{\mathbf{s}}_{2}$ characterize the electric fields of these waves. Four ports, $1 \mathrm{U}, 2 \mathrm{U}, 1 \mathrm{~L}$ and $2 \mathrm{~L}$, carrying incoming and outgoing electromagnetic waves, are used to represent these two polarizations above and below the system. The ports $1 \mathrm{U}$ and $2 \mathrm{U}$ respectively depict the polarizations $\hat{\mathbf{s}}_{\mathbf{1}}$ and $\hat{s}_{2}$ of the incoming or outgoing waves above the structure. Similarly, the ports $1 \mathrm{~L}$ and $2 \mathrm{~L}$ depict the polarizations $\hat{\mathbf{s}}_{\mathbf{1}}$ and $\hat{\mathbf{s}}_{\mathbf{2}}$ of the waves below the structure.

Mathematically, the incoming and outgoing waves of any system are related to each other by the system's scattering matrix containing frequency-dependent transmission and reflection coefficients. The total scattering matrix of the system can be written as the sum of two scattering matrices featuring the two types of interaction between the incoming and outgoing waves: (i) the resonance-assisted interaction (denoted by the $\tilde{\omega}$ channel in Figure 3), where the waves couple through the resonant modes of the structure, and (ii) the nonresonant interaction (denoted by $C$ in the figure), where the waves couple directly through a nonresonant background process.
The resonant interaction, as illustrated in the figure, is related to the mode's radiated electric field values at ports $1 \mathrm{U}, 2 \mathrm{U}, 1 \mathrm{~L}$, and $2 \mathrm{~L}$, which can be described by a scattering eigenvector $\mathbf{b}=$ $\left[\begin{array}{llll}b_{1 \mathrm{U}} & b_{2 \mathrm{U}} & b_{1 \mathrm{~L}} & b_{2 \mathrm{~L}}\end{array}\right]^{\mathrm{T}}$ containing the far-field components of quasinormal modes at each port. We characterize the nonresonant coupling by the frequency-dependent directprocess scattering matrix ${ }^{33}$, which has a nonchiral and polarization-independent response as

$$
C(\omega)=\left[\begin{array}{cccc}
r(\omega) & 0 & \mathrm{i} t(\omega) & 0 \\
0 & r(\omega) & 0 & \mathrm{i} t(\omega) \\
\mathrm{i} t(\omega) & 0 & r(\omega) & 0 \\
0 & \mathrm{i} t(\omega) & 0 & r(\omega)
\end{array}\right]
$$

$C$ must be unitary and symmetric ${ }^{34}$ for reciprocal materials. The frequency-dependent coefficients $r$ and $t$ are the direct reflection and transmission coefficients, respectively (the arbitrary phase factor of this matrix, which depends on the choice of the positions of our reference ports, ${ }^{35}$ can be omitted without any loss of generality, and we can suppose $r$ and $t$ to be positive real amplitudes such that $r^{2}+t^{2}=1$ ).

As we have recently shown, ${ }^{36}$ building upon ref 34 , it is possible to obtain the complete scattering matrix for any photonic system from the far-field properties of its eigenmodes. For a system with a set of complex eigenfrequencies $\tilde{\omega}_{j}(j=1, \ldots$, $m$ ), the scattering matrix can be written as

$$
S(\omega)=C(\omega)+\mathrm{i} \sum_{j=1}^{m} a_{j} \frac{\mathbf{b}_{j} \mathbf{b}_{j}^{T}}{\omega-\tilde{\omega}_{j}}
$$

where $a$ is a complex coefficient that can be calculated for a unitary and symmetric scattering matrix by imposing timereversal symmetry and reciprocity conditions. The general expression for coefficient $a$ is discussed in detail in ref 36 .

For our structure, the nonresonant reflection and transmission coefficients in eq 2 can be obtained by fitting ${ }^{37}$ the background response of the numerical simulation data to the response of a slab-like structure with an effective homogeneous permittivity and thickness. The calculated slowly varying transmission and reflection intensities $T=t^{2}$ and $R=r^{2}$ are shown as cyan and gray solid lines, respectively, in Figure 2. Using eq 3 , we predict various transmittances $T_{x x}, T_{y x}, T_{x y}$, and $T_{y y}$ as well as the AT for the example structure. The predicted quantities are represented by solid lines in Figure $2 \mathrm{a}$ and $\mathrm{b}$ and correspond very well with the simulation data.

Since we are able to predict the AT for the structure only from the complex frequencies and the far-field properties of the eigenmodes of the structure, it is now clear that AT is a resonant phenomenon that completely depends on the far-field characteristics. So, by tuning the mode properties, we can enhance the AT offered by the structure. It is instructive to study what the maximum AT is that can be achieved in any structure and how to design structures that can reach such a limit.

\section{ON THE LIMIT OF AT}

Dependence of the AT on the far-field properties of the eigenmodes stems from the interaction of the incoming and outgoing waves with the scattering eigenvector $\mathbf{b}$ of the modes. The eigenvector $\mathbf{b}$ can be normalized arbitrarily, as the scattering matrix (eq 3 ) is independent of such normalization, 
absorbing it in the evaluation of the coefficient $a_{j .}{ }^{36}$ Thus, for each mode, $\mathbf{b}$ basically comprises a set of field values $\left(b_{1 \mathrm{U}}, b_{2 \mathrm{U}}\right.$, $b_{1 \mathrm{~L}}$, and $\left.b_{2 \mathrm{~L}}\right)$. The field values $b_{1 \mathrm{U}}$ and $b_{2 \mathrm{U}}$ represent the two components of eigenmode polarization above the structure, and $b_{1 \mathrm{~L}}$ and $b_{2 \mathrm{~L}}$ represent the two eigenmode polarization components below the structure in the far field. For the example structure, the eigenmode field polarizations above and below the structure are related to each other by certain symmetry properties. The symmetry in the chosen structure is such that it returns to the original configuration after a series of operations: (i) an inversion along $z$, (ii) an inversion along $x$, and (iii) a clockwise rotation of $90^{\circ}$ (see the SI for details). This symmetry dictates that the eigenvectors of the system must satisfy the following relation among the polarization components of the eigenmode field below the structure and the polarization components above the structure:

$$
b_{1 \mathrm{~L}}= \pm b_{2 \mathrm{U}}, \quad b_{2 \mathrm{~L}}= \pm b_{1 \mathrm{U}}
$$

Inspection of calculated eigenfields from the FEM simulation confirms these relationships. In the range of frequencies we plot in Figure 2, it can be observed that the highest AT occurs around an isolated mode at $\omega_{0} / 2 \pi=364 \mathrm{THz}$. We can represent the far-field polarization $\left(b_{1 \mathrm{U}}, b_{2 \mathrm{U}}\right)$ of the eigenmode in terms of a set of normalized Stokes parameters $S_{1}, S_{2}$, and $\mathrm{S}_{3}{ }^{38}$

Expressing $\mathbf{b}$ in terms of the polarization parameters and applying eqs 1 and 3, we get an equation for the AT provided by a single mode in terms of its polarization parameters (as elaborated in the SI). We find that, at the eigenfrequency, the peak value of AT and the polarization parameters of the eigenmode have a direct relation:

$$
\text { AT }=\left|b_{1 U}\right|^{2}-\left|b_{2 U}\right|^{2}=\left|S_{1}\right|
$$

where $S_{1}$ is the first normalized Stokes parameter. This very simple, yet powerful relation holds a huge potential for obtaining the maximum possible AT by parametric optimization of any structure. A chosen structural parameter can be swept while tracking the isolated modes, and the calculated $S_{1}$ can predict the AT for each case. To illustrate this, we vary a single parameter, the total thickness of the chiral photonic crystal, and depict the predicted AT for the considered isolated eigenmode, as a function of the thickness. The blue circles in Figure 4a represent the theoretically predicted AT (the Stokes parameter $S_{1}$ ) for different thicknesses. The transmission simulation results are shown alongside (as black circles) to verify the prediction. The minor differences between the predicted and calculated values could be due to a small uncertainty in determining the far field of eigenmodes or a small contribution of a relatively distant mode. It can be seen that the optimum thickness is $0.5 a$, where $a$ is the periodicity of the structure. In fact, the structure we present in Figure 2 is the optimized case. It is to be noted that the maximum AT offered by the structure is $84 \%$, which is less than unity.

According to eq 5 , we would attain unity AT when $\left|S_{1}\right|=1$, i.e., when the eigenmode of the structure is completely linearly polarized along one of the $x$ and $y$ Cartesian axes of the structure. However, we find that the polarization parameters of the mode are restricted such that it is not always possible to get a mode that is completely linearly polarized in the far field. This constraint arises from the principle of reciprocity, which relates the polarization parameters of the resonant mode to the nonresonant scattering properties of the structure. In the following, we show how the principle of reciprocity puts a
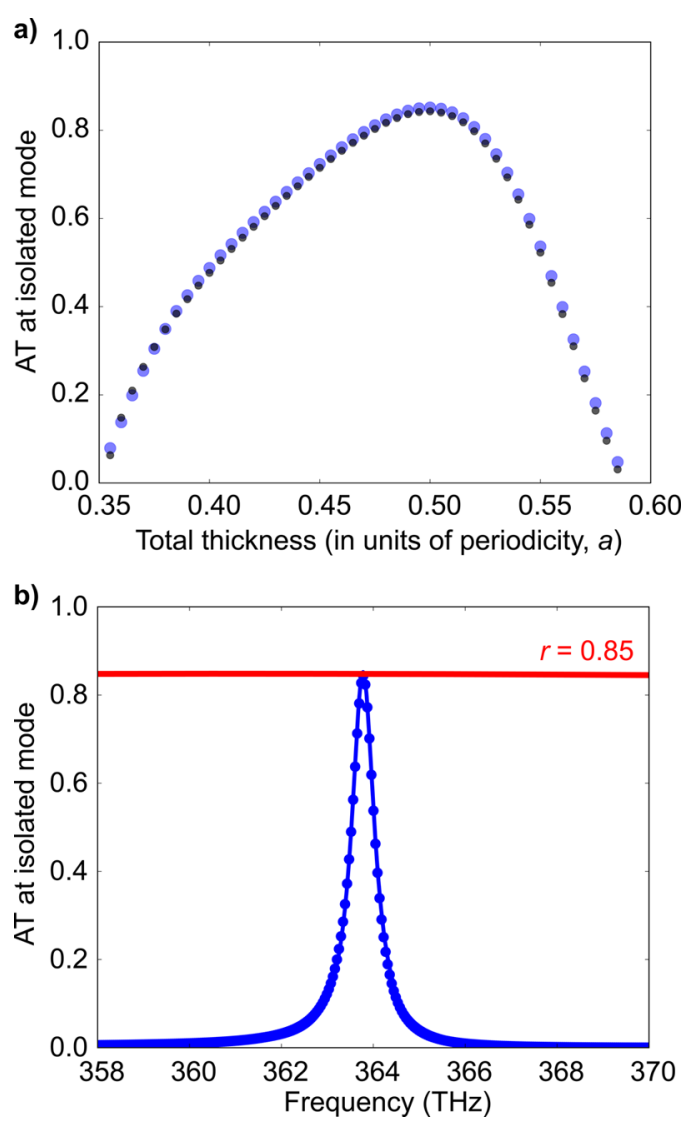

Figure 4. Parameter optimization and the fundamental limit of AT for a single mode of the proposed structure. (a) Predicted ATs for an isolated single mode for different thicknesses of the structure are shown as blue circles. Corresponding simulation results are shown as black small circles. (b) Calculated AT (blue solid line) and fundamental limit of maximum AT (red solid line) for a single mode together with FEM simulation data (blue data points).

fundamental limit on the possible polarization parameters for a mode, and hence the AT.

Following ref 34, the principle of reciprocity in coupledmode theory can be expressed for a single mode as

$$
C \mathbf{b}^{*}=-e^{\mathrm{i} \xi} \mathbf{b}
$$

where the total phase factor $\xi$ subsumes the arbitrary phase factors in the definition of $C$ and in the normalization of $\mathbf{b}$ (see Section 2 in the SI). Expressing eq 6 in terms of the components $r$ and $t$ that make up $C$ and the polarization parameters from the normalized $\mathbf{b}$ (as detailed in the SI), we obtain that eq 6 implies the relation

$$
\frac{t}{r}=\left|\frac{S_{3}}{S_{1}}\right|
$$

which indicates that the ratio between the Stokes parameters $S_{3}$ and $S_{1}$ of the eigenmode is related to the nonresonant background transmission of the structure at the mode frequency. This is extremely interesting, because it shows that a mode can be linearly polarized $\left(\left|S_{3}\right|=0,\left|S_{1}\right|=1\right)$ only if the corresponding nonresonant transmission coefficient $t$ is zero. Realistically, the background transmission from a homogeneous effective dielectric slab can never go to zero. Consequently, the modes of the system are generally constrained to be elliptically polarized. 
We use eq 7 to derive the fundamental constraint on the AT for the structure. The relation $S_{1}^{2}+S_{3}^{2} \leq 1$, which follows directly from the normalization of Stokes parameters, then implies $S_{1}^{2}\left(1+\frac{t^{2}}{r^{2}}\right) \leq 1$, and from eq 5 we obtain

$$
\mathrm{AT} \leq r=\sqrt{R}
$$

where $R=r^{2}$ is the nonresonant reflectivity of the structure. It is interesting to note that $\mathrm{AT}$, which is the difference between two resonant transmission intensities, has a limit that is given by the nonresonant reflection amplitude. For practical applications that require maximum $\mathrm{AT}$, it is thus important to engineer maximum nonresonant $R$ at the operation wavelength.

We choose the mode $\left(\omega_{0} / 2 \pi=364 \mathrm{THz}\right)$ having the highest AT from the FEM simulation data and calculate the fundamental limit for the maximum AT around the mode. The data points in Figure $4 \mathrm{~b}$ shows the FEM simulation-based $\mathrm{AT}$ as a function of the frequency of the normally incident light. The calculated fundamental limit, $r$, is shown as the red solid line. It can be seen that the theoretically calculated AT (blue solid line) as well as the FEM simulation data are very close to the fundamental limit.

The discussion so far in this section has been limited to the particular structure we proposed as an example. The specific symmetry properties of the structure were used to derive the results stated above. It is therefore important to verify the universality of the results on the limit of AT. In the following, we seek a fundamental limit for the AT offered by any singlemode resonator regardless of its symmetry properties and find that the result stays the same for the general case as well.

For deriving the limits for a general structure, we are no longer allowed to use the relation given by eq 4 , as it was derived from the specific symmetry properties of the example. Equation 6, which is based on the principle of reciprocity, is still valid and can be used to relate below-the-structure polarization parameters to the polarization parameters above the structure. Using this new relation in eq 3 leads to a general expression for AT in terms of the polarization parameters $S_{1}$ and $S_{3}$, the phase factor $\xi$, and the coefficients $r$ and $t$ :

$$
\mathrm{AT}=\left|\frac{r t^{2}\left(1-S_{1}^{2}\right) \sin \phi \sin \xi}{\left(1+r\left(\cos \phi \cos \xi+S_{1} \sin \phi \sin \xi\right)\right)^{2}}\right|
$$

where $\phi=\arctan \left(\frac{s_{3}}{S_{2}}\right)$ is the phase difference between the orthogonal polarization components of the eigenmode field above the structure. Please note that $r$ and $t$ are positive real coefficients here as a result of our convention for the arbitrary port phases such that $C$ takes the form of eq 2 . In the most general case, they thus represent the absolute value of the nonresonant reflection and transmission coefficients, respectively (see the SI for detailed derivation). The local maximum of this equation can be found from an analysis of its first-order derivatives and corresponds to the condition $\left|S_{1}\right|=r,\left|S_{3}\right|=t$, and $\xi= \pm \frac{\pi}{2}$. The corresponding value of $\mathrm{AT}$ at the maximum is $\mathrm{AT}_{\max }=r=\sqrt{ } R$. As we will now show, this maximum is a global maximum, which means that the nonresonant reflectivity puts the same fundamental limit for the AT in any single-mode resonator irrespective of its structural symmetry.

Using eq 9, we calculate AT for an isolated mode for 100000 randomly chosen points in the polarization space, i.e., random $S_{1}, \phi$, and $\xi$. The calculated AT is plotted in Figure 5 as a function of reflection coefficient $r$. It can be observed that all

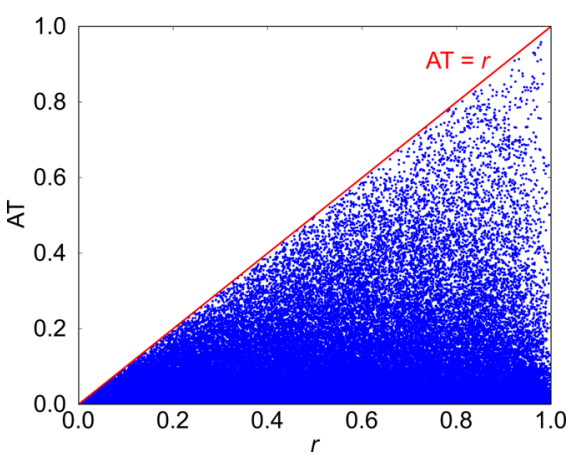

Figure 5. Fundamental limit of AT: Scatterplot of AT vs the nonresonant reflection coefficient $r$ for 100000 random eigenmode polarizations in a structure without any specific symmetry properties. Solid red line corresponds to $\mathrm{AT}=r$.

the values are below or at the predicted fundamental limit, $r$. It must also be noted that most of the random polarizations exhibit an AT significantly lower than the fundamental limit. Only very few combinations of polarization parameters can produce an AT close to the fundamental limit.

As we have shown using eq 7 and Figure $4 a$ earlier in this section, with an educated choice of structural symmetry and a proper optimization of structural parameters, we can now design structures that offer an AT near the fundamental limits. The structure shown as an example in the beginning of this paper would be an excellent candidate for realizing high AT with a fully dielectric structure.

\section{DISCUSSION AND CONCLUSIONS}

The basis for the outlined theoretical results is coupled-mode theory, which allows extending these results to any general resonator system. The discussed examples used for deriving the limits are dielectric 2-D periodic structures. For metallic or lossy systems including systems with more than two input/ output channels, the expression for the scattering matrix and the subsequent equations for AT and its limits can easily be rederived considering the nonradiative decay rates of the modes. ${ }^{36}$ It must be noted that it is possible to use the coupledmode theory for describing the scattering properties of arbitrary-shaped particles. ${ }^{39}$ This allows us to extend our formalism to such particles as well.

The presented simulation results consider a 2-D infinitely extended periodic structure and transmission of normally incident light. We note that AT for such a geometry is expected to vary as a function of the angle of incidence due to the Bloch eigenmode's dispersion. A finite numerical aperture (NA) of the experimental setup should be taken into account for experimental realization of the results, as we do for our example system in the SI.

In conclusion, we showed that the AT in chiral resonators depends strongly on the far-field properties of their eigenmodes. We developed a theoretical formalism that can predict AT offered by any system from its complex eigenmodes. We investigated the theoretical maximization of AT in chiral resonators. A fundamental limit for AT provided by a single mode is presented. We also proposed the design for a chiral photonic crystal that can offer AT as high as the fundamental limit, which is the nonresonant reflection amplitude of the system. The theoretical formalism presented here opens ways for designing and optimizing new structures for light manipulation. 


\section{ASSOCIATED CONTENT}

\section{S Supporting Information}

The Supporting Information is available free of charge on the ACS Publications website at DOI: 10.1021/acsphotonics.6b00947.

$$
\text { Additional information (PDF) }
$$

\section{AUTHOR INFORMATION}

\section{Corresponding Author}

*E-mail: verhagen@amolf.nl.

\section{ORCID}

Ewold Verhagen: 0000-0002-0276-8430

\section{Notes}

The authors declare no competing financial interest.

\section{ACKNOWLEDGMENTS}

This work is part of the research program of The Netherlands Organization for Scientific Research (NWO). The authors acknowledge the support from the European Research Council (ERC) and an industrial partnership program between Philips and NWO.

\section{REFERENCES}

(1) Vallius, T.; Jefimovs, K.; Turunen, J.; Vahimaa, P.; Svirko, Y. Optical Activity in Subwavelength-Period Arrays of Chiral Metallic Particles. Appl. Phys. Lett. 2003, 83, 234-236.

(2) Papakostas, A.; Potts, A.; Bagnall, D. M.; Prosvirnin, S. L.; Coles, H. J.; Zheludev, N. I. Optical Manifestations of Planar Chirality. Phys. Rev. Lett. 2003, 90, 107404.

(3) Kuwata-Gonokami, M.; Saito, N.; Ino, Y.; Kauranen, M.; Jefimovs, K.; Vallius, T.; Turunen, J.; Svirko, Y. Giant Optical Activity in Quasi-Two-Dimensional Planar Nanostructures. Phys. Rev. Lett. 2005, 95, 227401.

(4) Barnes, W. L.; Dereux, A.; Ebbesen, T. W. Surface Plasmon Subwavelength Optics. Nature 2003, 424, 824-830.

(5) Novotny, L.; van Hulst, N. Antennas for Light. Nat. Photonics 2011, 5, 83-90.

(6) Fan, S.; Winn, J. N.; Devenyi, A.; Chen, J. C.; Meade, R. D.; Joannopoulos, J. D. Guided and Defect Modes in Periodic Dielectric Waveguides. J. Opt. Soc. Am. B 1995, 12, 1267-1272.

(7) Pendry, J. B.; Schurig, D.; Smith, D. R. Controlling Electromagnetic Fields. Science 2006, 312, 1780-1782.

(8) Yu, N.; Capasso, F. Flat Optics with Designer Metasurfaces. Nat. Mater. 2014, 13, 139-150.

(9) Watts, M. R.; Haus, H. A. Integrated Mode-Evolution-Based Polarization Rotators. Opt. Lett. 2005, 30, 138-140.

(10) Bayat, K.; Chaudhuri, S. K.; Safavi-Naeini, S. Ultra-Compact Photonic Crystal Based Polarization Rotator. Opt. Express 2009, 17, 7145 .

(11) Yu, N.; Aieta, F.; Genevet, P.; Kats, M. A.; Gaburro, Z.; Capasso, F. A Broadband, Background-Free Quarter-Wave Plate Based on Plasmonic Metasurfaces. Nano Lett. 2012, 12, 6328-6333.

(12) Lin, D.; Fan, P.; Hasman, E.; Brongersma, M. L. Dielectric Gradient Metasurface Optical Elements. Science 2014, 345, 298-302.

(13) Shen, B.; Wang, P.; Polson, R.; Menon, R. An IntegratedNanophotonics Polarization Beamsplitter with $2.4 \times 2.4$ um2 Footprint. Nat. Photonics 2015, 9, 378-382.

(14) Fedotov, V. A.; Mladyonov, P. L.; Prosvirnin, S. L.; Rogacheva, A. V.; Chen, Y.; Zheludev, N. I. Asymmetric Propagation of Electromagnetic Waves Through a Planar Chiral Structure. Phys. Rev. Lett. 2006, 97, 167401.

(15) Zhou, H.; Zhen, B.; Hsu, C. W.; Miller, O. D.; Johnson, S. G.; Joannopoulos, J. D.; Soljačić, M. Perfect Single-Sided Radiation and Absorption Without Mirrors. Optica 2016, 3, 1079.
(16) Konishi, K.; Nomura, M.; Kumagai, N.; Iwamoto, S.; Arakawa, Y.; Kuwata-Gonokami, M. Circularly Polarized Light Emission from Semiconductor Planar Chiral Nanostructures. Phys. Rev. Lett. 2011, 106, 057402.

(17) le Feber, B.; Rotenberg, N.; Kuipers, L. Nanophotonic Control of Circular Dipole Emission. Nat. Commun. 2015, 6, 6695.

(18) Bliokh, K. Y.; Rodríguez-Fortuño, F. J.; Nori, F.; Zayats, A. V. Spin-Orbit Interactions of Light. Nat. Photonics 2015, 9, 796-808.

(19) Tang, Y.; Cohen, A. E. Enhanced Enantioselectivity in Excitation of Chiral Molecules by Superchiral Light. Science 2011, 332, 333-336.

(20) Schwanecke, A. S.; Fedotov, V. A.; Khardikov, V. V.; Prosvirnin, S. L.; Chen, Y.; Zheludev, N. I. Nanostructured Metal Film with Asymmetric Optical Transmission. Nano Lett. 2008, 8, 2940-2943.

(21) Drezet, A.; Genet, C.; Laluet, J.-Y.; Ebbesen, T. W. Optical Chirality Without Optical Activity: How Surface Plasmons Give a Twist to Light. Opt. Express 2008, 16, 12559.

(22) Singh, R.; Plum, E.; Menzel, C.; Rockstuhl, C.; Azad, A. K.; Cheville, R. A.; Lederer, F.; Zhang, W.; Zheludev, N. I. Terahertz Metamaterial with Asymmetric Transmission. Phys. Rev. B: Condens. Matter Mater. Phys. 2009, 80, 153104.

(23) Plum, E.; Fedotov, V. A.; Zheludev, N. I. Planar Metamaterial with Transmission and Reflection That Depend on the Direction of Incidence. Appl. Phys. Lett. 2009, 94, 131901.

(24) Konishi, K.; Bai, B.; Meng, X.; Karvinen, P.; Turunen, J.; Svirko, Y. P.; Kuwata-Gonokami, M. Observation of Extraordinary Optical Activity in Planar Chiral Photonic Crystals. Opt. Express 2008, 16, 7189.

(25) Meng, X.; Bai, B.; Karvinen, P.; Konishi, K.; Turunen, J.; Svirko, Y.; Kuwata-Gonokami, M. Experimental Realization of All-Dielectric Planar Chiral Metamaterials with Large Optical Activity in Direct Transmission. Thin Solid Films 2008, 516, 8745-8748.

(26) Menzel, C.; Rockstuhl, C.; Lederer, F. Advanced Jones Calculus for the Classification of Periodic Metamaterials. Phys. Rev. A: At., Mol., Opt. Phys. 2010, 82, 053811.

(27) Menzel, C.; Helgert, C.; Rockstuhl, C.; Kley, E.-B.; Tünnermann, A.; Pertsch, T.; Lederer, F. Asymmetric Transmission of Linearly Polarized Light at Optical Metamaterials. Phys. Rev. Lett. 2010, 104, 253902.

(28) Mutlu, M.; Akosman, A. E.; Serebryannikov, A. E.; Ozbay, E. Diodelike Asymmetric Transmission of Linearly Polarized Waves Using Magnetoelectric Coupling and Electromagnetic Wave Tunneling. Phys. Rev. Lett. 2012, 108, 213905.

(29) Li, Z.; Gokkavas, M.; Ozbay, E. Manipulation of Asymmetric Transmission in Planar Chiral Nanostructures by Anisotropic Loss. Adv. Opt. Mater. 2013, 1, 482-488.

(30) Liu, D.-j.; Xiao, Z.-y.; Ma, X.-1.; Wang, Z.-h Broadband Asymmetric Transmission and Multi-Band $90^{\circ}$ Polarization Rotator of Linearly Polarized Wave Based on Multi-Layered Metamaterial. Opt. Commun. 2015, 354, 272-276.

(31) Kenanakis, G.; Xomalis, A.; Selimis, A.; Vamvakaki, M.; Farsari, M.; Kafesaki, M.; Soukoulis, C. M.; Economou, E. N. ThreeDimensional Infrared Metamaterial with Asymmetric Transmission. ACS Photonics 2015, 2, 287-294.

(32) COMSOL Multiphysics 5.2. http://www.comsol.com.

(33) Suh, W.; Wang, Z.; Fan, S. Temporal Coupled-Mode Theory and the Presence of Non-Orthogonal Modes in Lossless Multimode Cavities. IEEE J. Quantum Electron. 2004, 40, 1511-1518.

(34) Fan, S.; Suh, W.; Joannopoulos, J. D. Temporal Coupled-Mode Theory for the Fano Resonance in Optical Resonators. J. Opt. Soc. Am. A 2003, 20, 569-572.

(35) Wang, K. X.; Yu, Z.; Sandhu, S.; Fan, S. Fundamental Bounds on Decay Rates in Asymmetric Single-Mode Optical Resonators. Opt. Lett. 2013, 38, 100-102.

(36) Alpeggiani, F.; Nikhil, P.; Verhagen, E.; Kuipers, L. Quasinormal-Mode Expansion of the Scattering Matrix. arXiv:1609.03902 2016.

(37) Fan, S.; Joannopoulos, J. D. Analysis of Guided Resonances in Photonic Crystal Slabs. Phys. Rev. B: Condens. Matter Mater. Phys. 2002, 65, 235112. 
(38) Born, M.; Wolf, E. Principles of Optics: Electromagnetic Theory of Propagation, Interference and Diffraction of Light; CUP Archive, 2000. (39) Ruan, Z.; Fan, S. Temporal Coupled-Mode Theory for Light Scattering by an Arbitrarily Shaped Object Supporting a Single Resonance. Phys. Rev. A: At., Mol., Opt. Phys. 2012, 85, 043828. 